\title{
Pronóstico de accidentes viales en el espacio intrametropolitano de Toluca: un enfoque Bayesiano
}

\author{
Raquel Hinojosa Reyes ${ }^{1}$, Carlos Felix Garrocho Rangel ${ }^{2}$, Juan Campos Alanís ${ }^{3}$ e \\ Araceli Consuelo Campero Carmona ${ }^{4}$
}

\begin{abstract}
Resumen: En años recientes la explicación de los accidentes de tránsito tiende a enfocarse en la estructura espacial urbana, tal explicación se basa en ciertas características del entorno como son: el uso de suelo, los sistemas viales, actividades económicas, población e intensidad de flujos vehiculares, entre otros. En el círculo de investigadores de este fenómeno, dichos factores se clasifican como indirectos y favorecen la ocurrencia de accidentes de tránsito en las ciudades del mundo. Este artículo se enfoca a realizar un análisis de riesgo de accidentes viales en la zona intrametropolitana de Toluca a nivel de AGEB . Se abordan los accidentes viales de tipo colisión. La investigación gira en torno a la identificación de los factores de la estructura urbana que más afectan el riesgo de accidentes de tránsito, lo que demarca un filtro estadístico para potenciar las variables a considerar en tres modelos con enfoque Bayesiano: Poisson-Gamma bivariante, multivariante y probabilístico Binomial Negativo. Los resultados indican que el flujo vehicular y la densidad de empleo son los indicadores que más se relacionan con los accidentes de tránsito tipo colisión en la zona.
\end{abstract}

Palabras-clave: accidentes de tránsito en ciudades, estructura urbana, modelos Bayesianos.

\begin{abstract}
In recent years, the explanation of traffic accidents tended to focus on the urban spatial structure. It is considered that certain features of the environment (e.g. land use, road system, economic activities, population and intensity of vehicular flows) are indirect factors that favor the occurrence of collision traffic accidents in urban areas. The central part of the intrametropolitan zone of Toluca in Mexico) records elevated rates of traffic accidents. This research aims to identify the physical elements of the urban structure, which affects most of the risks about traffic accidents, in the study area, at AGEB level (which stands for the Basic Geo-statistical Area). Three Bayesian models are being used in order to achieve the research objective: Bivariant Poisson-Gamma, Multivariate and Negative Binomial probabilistic. The results indicate that vehicular flows and employment density are the most related variables linked to the risk of collision traffic accidents, in the study area.

Keywords: Traffic accidents in cities, urban structure, Bayesian models.
\end{abstract}

\section{INTRODUCCIÓN}

Las ciudades como Toluca en el Estado de México y otras de Latinoamérica son sistemas complejos en constante transformación social, económica, en infraestructura y normalmente registran crecimiento demográfico acelerado y fuerte expansión en su periferia (Negrete, 2002; Garza, 2009). Esto se traduce, en serios congestionamientos de tráfico en su núcleo central, que normalmente es el nodo de actividades más importante de la ciudad (De Mattos, 2002; Garrocho \& Flores, 2009). En un entorno de crecimiento urbano tan dinámico se generan ciudades poco ordenadas, con notables problemas de organización espacial y de movilidad (Garrocho, 2013).-El automóvil es considerado uno de los principales elementos perturbadores de la vida ciudadana (Chueca, 1997), genera problemas de tráfico, aumento exponencial del número de desplazamientos, contaminación, ruido, y accidentes de tránsito (Graizbord, 2008; Alcántara 2010). Los accidentes de tránsito son un problema que aqueja a las concentraciones urbanas de todo el mundo.

\footnotetext{
${ }^{1}$ Raquel Hinojosa Reyes, Facultad de Geografía

Universidad Autónoma del Estado de México. (r_hinojosa28@hotmail.com)

2 Carlos Felix Garrocho Rangel, Colegio Mexiquense.

(cfgarrocho@gmail.com)

3 Juan Campos Alanís, Facultad de Geografía

Universidad Autónoma del Estado de México.

(juan campos70@hotmail.com)

${ }_{4}^{4}$ Araceli Consuelo Campero Carmona, Facultad de Ingeniería

Universidad Autónoma del Estado de México. (arkeli9502@yahoo.com.mx)

Manuscrito recebido em 09/01/2015 e aprovado para publicação em 01/06/2015.

Este artigo é parte de TRANSPORTES v. 23, n. 2, 2015. ISSN: 2237-1346 (online). DOI: 10.14295/transportes.v23i2.882
}

La mayoría, no importa su tipo, ocurren en áreas urbanas (Kim et al., 2006; Boher, 2006), sin embargo, su frecuencia varía en el espacio en función de ciertas variables reportadas en la literatura. Así, los estudios de accidentalidad a nivel de agregación macro intentan develelar cómo ciertos elementos del entorno, inciden en la ocurrencia de accidentes (Christie, 2000; Glaister \& Graham, 2005; Noland \& Quddus, 2003; Kim et al., 2006, Quddus, 2008).

Estos elementos del entorno por lo regular son componentes físicos de la estructura urbana, que explican en términos estadísticos un accidente vehicular. Entre los principales componentes, se reporta el uso del suelo, la intensidad o tipo de actividades económicas que se ubican en ciertas zonas de la ciudad, las características socioeconómicas de la población y las características del tráfico (Fuentes \& Hernández, 2009; Clifton et al., 2009; Jones et al., 2008).

El objetivo de este trabajo es desarrollar un modelo estadístico macroscópico de tipo bayesiano que permita predecir la ocurrencia de accidentes de tránsito tipo colisión en el centro de la zona metropolitana de Toluca (ZMT), México. Este modelo debe generar resultados con una desagregación espacial a escala de AGEB, en función de las características de tráfico, sociales y de la estructura física de la zona de estudio (e.g. tipologías de uso de suelo, socioeconómicas y de la infraestructura vial). Se espera que los hallazgos sirvan de insumo para la generación de políticas urbanas y acciones puntuales orientadas a mejorar la seguridad vial y la planificación del uso del suelo (Agüero-Valverde \& Jovanis 2006).

Durante la última década diversos investigadores han considerado que los datos de accidentes de tránsito tienen 


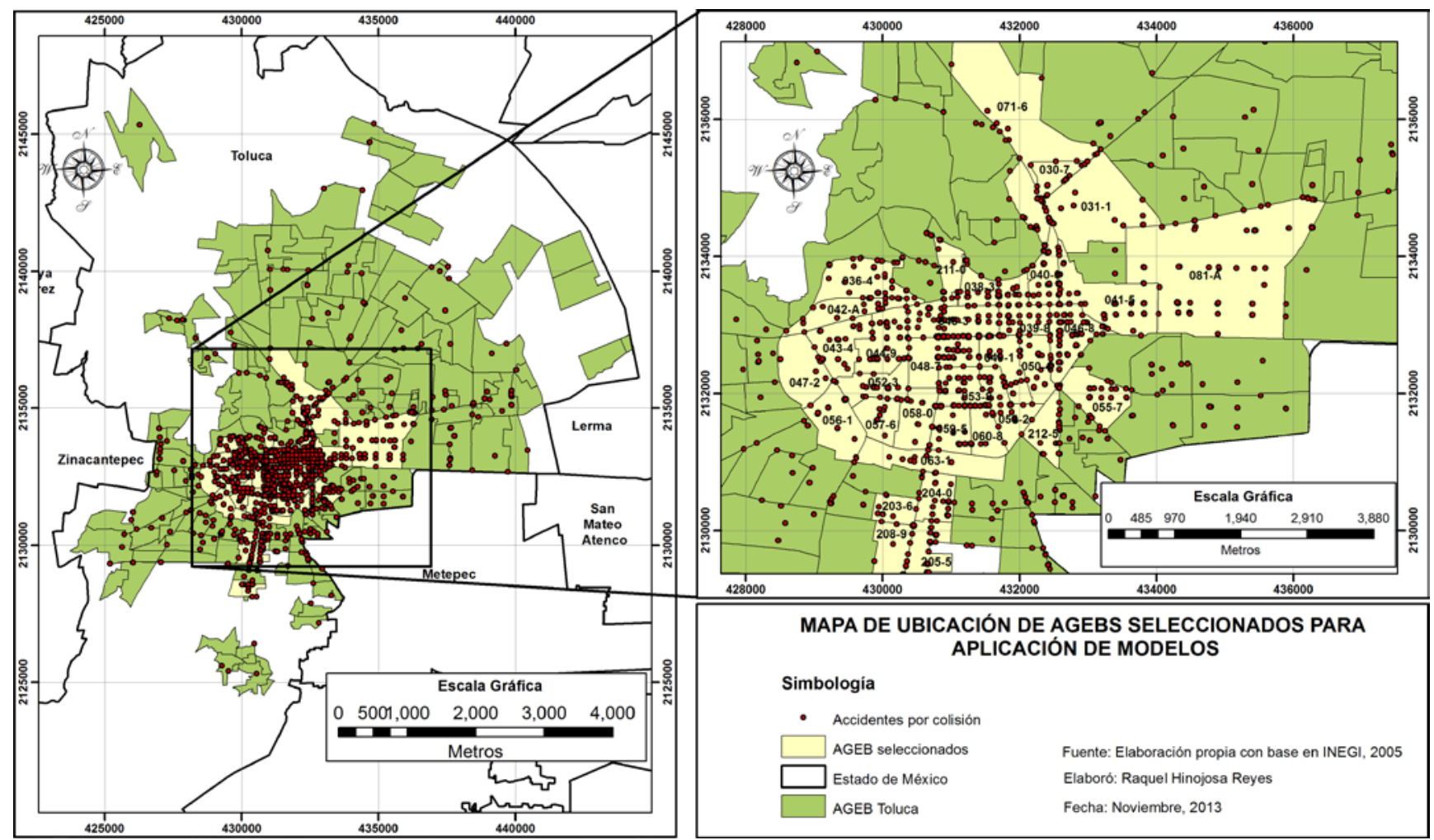

Figura 1. Ubicación y registro de accidentes en la zona de estudio

características propias y a partir de ellas se determina la manera como se pueden modelar. Tienen una localización espacial específica, lo que hace que estén relacionados con las características de su entorno, registran cierta frecuencia, tienen un componente aleatorio por lo tanto usualmente no son independientes ni estacionarios. Es por esto que suelen utilizarse modelos basados en el proceso de Poisson para explicar el comportamiento estadístico de los accidentes viales urbanos, (Hadayeghi et al., 2003; Miaou \& Lord, 2003; Kim et al., 2006; Quddus, 2008; Pulugurtha et al., 2012).

El paradigma alternativo para inferir datos estadísticos referentes ha accidentes de tránsito es el enfoque Bayesiano, mismo que considera la dependencia (o independencia) espacio-temporal de las variables y los eventos (Miaou \& Lord, 2003; Hadayeghi et al., 2003; Miaou \& Jin, 2005; Miranda et al., 2005; Quddus, 2008; Siddiqui et al., 2012).

El texto se estructura en cuatro apartados. En el primero se presenta el escenario de información, que incluye el área de estudio y el origen y características de los datos utilizados; en el segundo apartado se explican brevemente los modelos utilizados; para posteriormente, en el apartado tres, muestra la aplicación y los resultados comparando los tres modelos seleccionados (Poisson-Gamma bivariante, Poisson-Gamma multivariante y probabilístico Binomial Negativo), y finalmente las conclusiones.

\section{ESCENARIO DE INFORMACIÓN}

La zona de estudio se delimitó en función del registro georreferenciado de accidentes vehiculares por $A G E B$, por lo que el estudio se enfoca al centro tradicional de negocios de la Zona Intrametropolitana de Toluca. Es el área de máximos flujos de tráfico vehicular y de movilidad peatonal en la ciudad, y ha sido delimitado funcionalmente en un trabajo previo (Garrocho \& Flores, 2009). La zona queda determinada por 35 AGEB que registran diez o más accidentes de tránsito tipo colisión, (Figura 1).
En la figura se observa el mapa elaborado a través de herramientas de Sistema de Información Geográfica (ArcGIS), que muestra el patrón de distribución de accidentes tipo colisión de forma puntual, los polígonos en color claro representan las $A G E B$ seleccionadas para análisis y las zonas verdes representan el resto de $A G E B$ que integran el área urbana.

\subsection{Presentación e inventario de información}

Los Accidentes de tránsito tipo colisión ( $\left.x_{i}\right)$, representan el 90\% de los 1856 registros para el año 2005 en el municipio de Toluca, fueron considerados por la presencia estadística que tienen en la zona. En este tipo de accidentes se nincluyen choques en ángulo recto, contra objeto fijo, de frente, lateral y por alcance. Los datos fueron proporcionados por la Dirección de Control de Tránsito de Toluca (DCTT) en formato de lista en un atchivo de hoja de cálculo (Excel), los cuales se georeferenciaron por intersección o tramo y fueron agregados por $A G E B$ (tarea realizada mediante un proceso de asignación espacial usando la herramienta ArcGIS) ver figura 2. La estadística descriptiva muestra un mínimo de 10 accidentes, un máximo de 91, el promedio de 33 y la desviación estándar de 20 accidentes por $A G E B_{i}$.

En la imagen que se muestra de lado izquierdo presenta los datos puntuales de los accidentes $x_{i}$ y de lado derecho la información agregada por $A G E B$, degradada por color, la tonalidad más oscura refiere a la mayor cantidad de accidentes $x_{i}$.

Antes de iniciar el proceso de análisis de información vial para el caso de la zona de estudio, fue necesario consultar las fuentes de información disponibles, con la finalidad de analizar la estructura y calidad de información existente. Se identificaron una serie de limitantes que se listan a continuación: registro parcial de accidentes para solo tres 


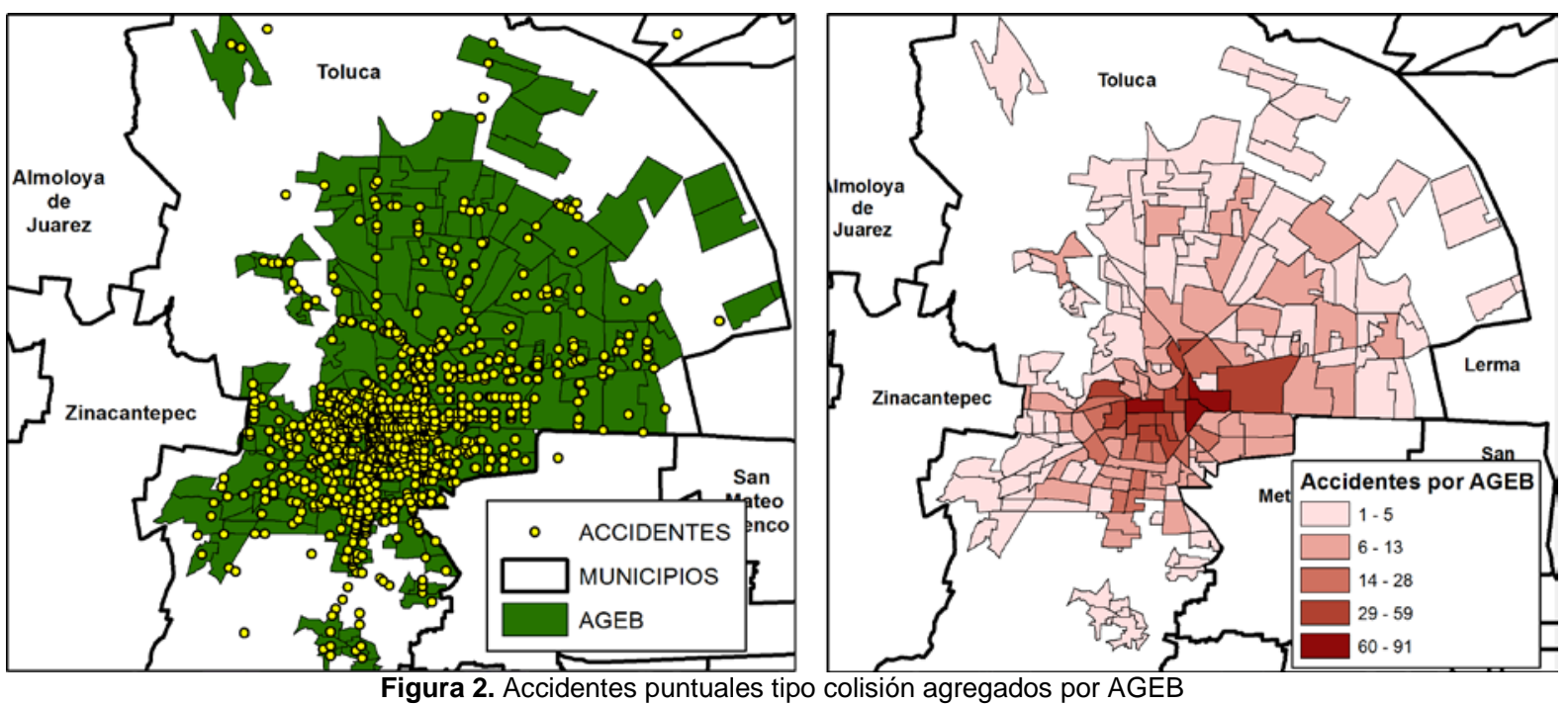

casos: cuando los daños materiales y físicos son de gravedad, cuando no hay un acuerdo entre los involucrados y cuando hay decesos. Fuentes de información multiple, existen al menos tres fuentes o instituciones que registran los accidentes y sus características, cada una de ellas posee un procedimiento determinado para recabar la información y por lo tanto, existe heterogeneidad en el número, calidad y cantidad de datos. Además existen barreras para el acceso a la información debido a que los organismos municipales y estatales administran de forma independiente la información colectada, la cual consideran de carácter privado, confidencial y de uso exclusivo para cada corporación, puesto que no la canalizan a una autoridad central ni tampoco tienen establecidos procedimientos para acceder a ella. En otras palabras, la información existente generalmente no está validada y es muy heterogénea debido a la ausencia de procedimientos sistemáticos.

Otros indicadores que explican el evento de acuerdo a la literatura (Peden et al., 2004; Noland \& Quddus, 2003; Gleister \& Graham, 2005; Clarke et al., 2006; Erso, 2006; Agüero-Valverde \& Quddus, 2008; Fuentes \& Hernández, 2009; Siddiqui et al., 2012) se clasificaron en cuatro categorías: flujo vehicular; características de las vialidades; características de uso de suelo y características socioeconómicas ver tabla 1.

Flujo Vehícular $\left(F v_{i}\right)$, es considerado uno de los factores más influyentes en la ocurrencia de accidentes de tránsito. La variable ideal sería el número de vehículos que transitan por cada $A G E B_{i}$. Sin embargo la información no está disponible, por lo tanto la alternativa es utilizar el total de vehículos registrados en cada $A G E B_{i}$, con la observación de que los vehículos registrados para una $A G E B_{i}$ pueden transitar en otras $A G E B_{i}$ vecinas. Para ello se utiliza un modelo de interacción espacial (Quddus, 2008), que permitió contabilizar el número de vehículos en cada $A G E B_{i}$. El modelo se puede apreciar en la ecuación (1) y expresa el cálculo del flujo vehicular en cada $A G E B_{i}$.

$$
F v_{i}=v_{i}+\sum_{j=1}^{n-1} \frac{v_{j}}{d_{i j}} i \neq j
$$

Donde:

$F v_{i}$ :es el total de vehículos que pueden estar transitando dentro de un $A G E B_{i}$; $v_{i}$ :representa los vehículos registrados por cada $A G E B_{i}$;

$v_{j}$ : son los vehículos registrados en la $A G E B_{j}$, $d_{i j}$ es la distancia central entre el $A G E B_{i}$ y la $A G E B_{j}$; $n$ :es el número total de $A G E B$.

Aplicación del modelo de interacción espacial. Para aplicar el modelo se realizó el siguiente proceso: primero, se obtuvo el centroide de cada $A G E B_{i}$ que contiene el dato de viviendas particulares que disponen de automóvil o camioneta propia. Segundo, con el uso del software transCAD se genera la matriz de distancias entre la $A G E B_{i}$ y la $A G E B_{j}$ para las n- $A G E B$ y tercero, se sistematiza en una hoja de cálculo Excel la aplicación del modelo que permite aproximar el flujo vehicular. La estimación resultante fue validada con datos de flujos vehiculares reales de algunas intersecciones de la ciudad de Toluca.

Características de vialidades. Esta variable se definió en función de la densidad de intersecciones ( $D$ int $_{i}$ ) y densidad de longitud vial por tipo: vias de primer orden $\left(D l v p_{i}\right)$, vías de segundo orden ( $D l v s_{i}$ ) y vías de tercer orden $\left(D l v t_{i}\right)$. Las $A G E B_{i}$ que contienen una longitud mayor de vialidades primarias y secundarias tienen más probabilidad de tener un accidente porque su flujo vehicular es mayor (Quddus, 2008).

Características de uso de suelo. Esta definida por la densidad de unidades económicas por sector ( Dues $_{i}$ ) se utilizó la información de la localización espacial de las unidades económicas (Denue, 2009) y se construyeron indicadores por sector de actividad: densidades relativas de usos de suelo comercial ( Dcomercial $_{i}$ ), de servicios privados ( Dservicios $_{i}$ ), servicios gubernamentales (Dservgub $b_{i}$ ), e industrial ( Dindustrial $_{i}$ ). La Densidad de población $\left(D p o b_{i}\right)$ y densidad de viviendas particulares ( $\operatorname{Dvivp}_{i}$ ) relativas al uso residencial, se obtuvieron del censo de población y vivienda (Inegi, 2005).

Características socio-económicas: Se obtuvieron del censo de población y vivienda (Inegi, 2005), a partir de registros de población: grupos de edad de 15 a 24 años ( $p p 1524_{i}$ ) y de 60 y más ( $p$ p60mas $_{i}$ ); empleo: densidad de empleo $\left(\right.$ Demp $\left._{i}\right)$ se calculó a partir del Denue (2009). 
Tabla 1. Indicadores

\begin{tabular}{|c|c|c|c|}
\hline Variable & Indicador & Media & $\begin{array}{l}\text { Desviación es- } \\
\text { tándar }\end{array}$ \\
\hline $\begin{array}{l}\text { Flujo } \\
\text { Vehicular }\end{array}$ & Flujo vehicular $F v_{i}$ & 98038625 & 11691139 \\
\hline \multirow{4}{*}{$\begin{array}{l}\text { Características } \\
\text { de vialidades }\end{array}$} & Densidad de intersecciones $D$ int $_{i}$, & 81 & 44 \\
\hline & Densidad de longitud vial primaria $D l v p_{i}$, & 78 & 90 \\
\hline & Densidad de longitud vial secundaría $D l v s_{i}$, & 3 & 2 \\
\hline & Densidad de longitud vial tercer orden $D l v t_{i}$, & 63 & 41 \\
\hline \multirow{7}{*}{$\begin{array}{l}\text { Características } \\
\text { de uso de suelo }\end{array}$} & Densidad de unidades económicas por $A G E B D_{u e s}$, & 9 & 8 \\
\hline & Densidad comercial Dcomercial $_{i}$ & 4 & 5 \\
\hline & Densidad de servicios Dservicios $_{i}$, & 3 & 2 \\
\hline & Densidad de servicios Gubernamentales Dservgub $b_{i}$, & 0.5 & 1 \\
\hline & Densidad Industrial Dindustrial $_{i}$ & 0.6 & 0.4 \\
\hline & Densidad de Población $D p o b_{i}$ & 81 & 33 \\
\hline & Densidad de viviendas particulares Dvivp $_{i}$, & 19 & 8 \\
\hline \multirow{5}{*}{$\begin{array}{l}\text { Características So- } \\
\text { cio-económicas }\end{array}$} & Población de 15 a 24 años $p p 1524_{i}$ & 18 & 2 \\
\hline & Población de 60 años y más pp60mas $_{i}$, & 9 & 3 \\
\hline & Densidad de Empleo $\operatorname{Demp}_{i}$ & 68 & 51 \\
\hline & Índice de Marginación $i m_{i}$, inverso $i b_{i}$ & 0.9 & 0.3 \\
\hline & 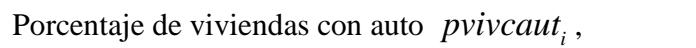 & 52 & 15 \\
\hline
\end{tabular}

Se agrupó la información por sector y subsector, se promedió el número de empleados por unidad económica y se dividió el total de empleos entre el área de la $A G E B_{i}$; índice de marginación $\left(\mathrm{im}_{i}\right.$ ) la marginación social es un fenómeno estructural que diagnostica la situación social con respecto a la distribución de la riqueza (Rodríguez, 2012). Este índice se obtuvo con información de Inegi (2005) y con la metodología del Consejo Nacional de Población (Conapo, 2005), finalmente se aplicó el inverso para tener el índice de bienestar $\left(i b_{i}\right)$. Viviendas con automóvil: esta representado por el porcentaje de viviendas con auto ( pvivcaut $_{i}$ ) dentro de una $A G E B_{i}$.

\subsection{Estandarización de información de indicadores}

Los indicadores se calcularon y se estandarizaron para obtener el valor normalizado de cada uno de ellos y poder análizarlos en conjunto en el modelo (Ecuación 2).

$$
z=\frac{x-\bar{x}}{\sigma}
$$

Donde

$x$ :reperesenta el valor observado de cada $A G E B_{i}$;

$\bar{x}$ :representa la media muestral del indicador; $y$

$\sigma$ :es la desviación estándar.

\subsection{Significancia de indicadores y métodos}

En este apartado se presenta la significancia de información con dos criterios diferentes: Primero se utiliza el método de componentes principales, donde se realiza un análisis multivariado que permite obtener un escalar sintético de un conjunto de indicadores y ayuda en la identificación de indicadores significativos al construir un modelo (Campos, 2009). Para verificar que los indicadores elegidos sean los adecuados, se considera el criterio de la varianza total explicada, que indica la proporción de la variabilidad del grupo de indicadores seleccionados, (Se considera que un grupo de variables es adecuado, cuando la suma acumulada en el segundo factor es superior a 50\%). Por otro lado, el segundo método referente a la matriz de correlación de Pearson, utiliza comúnmente una medida de la magnitud del efecto de una variable o un conjunto de variables independientes sobre una variable dependiente. Se considera que valores alrededor de 0.1 representan efectos bajos, valores alrededor de 0.3 representan efectos medios, valores ligeramente abajo o arriba de 0.5 representan efectos altos, y valores notablemente arriba de 0.5 representan efectos muy altos (Field, 2005: p. 112).

\subsection{Selección de los indicadores para usar en tres especificaciones de modelos}

\subsubsection{Primer filtro: componentes principales}

Inicialmente se analizaron 17 indicadores. Al aplicar el método de componentes principales el valor de la varianza acumulada hasta el segundo factor fue de 54.6\%, lo que permite afirmar que la selección de los indicadores es satisfactoria. Sin embargo, se realizaron otras pruebas eliminando los indicadores con baja representación: $\left(D_{v i v p_{i}}\right)$, $\left(D p o b_{i}\right),\left(p p 1524_{i}\right),\left(D l v p_{i}\right),\left(D l v t_{i}\right), y\left(D\right.$ int $\left._{i}\right)$. Como Dvivp $_{i}$ y Dpob $_{i}$ son un aproximado de la densidad de uso de suelo residencial.

Intuitivamente se esperaría que en las zonas de alta densidad se incrementen las interacciones potenciales entre vehículos. Sin embargo, los resultados para estas variables fueron negativos y poco significativos, lo que indica que en la medida que se incrementa la densidad de población y vi- 
Tabla 2. Indicadores para instrumentación de modelos

\begin{tabular}{l}
\hline \multicolumn{1}{c}{ Indicador } \\
\hline Flujo vehicular $F v_{i}$, \\
Densidad de empleo Demp \\
Densidad de longitud vial secundaría Dlvs $_{i}$, \\
Población de 60 años y más pp60mas \\
\hline
\end{tabular}

vienda disminuye el número de accidentes $x_{i}$. Este resultado es consistente con lo que reportan otros estudios (Fuentes \& Hernández, 2009; Kim et al., 2006; Pulugurtha et al., 2012). Una explicación posible es el comportamiento más prudente de los conductores en zonas de alta densidad (e.g. tienden a transitar a menor velocidad en estas áreas), así como la presencia de más y mejores controles de tráfico (e.g. señalética, topes, vigilancia), lo que reduce la posibilidad de accidentes $x_{i}$. Por su parte, la variable $p p 1524_{i}$ también es poco significativa para el modelo. No obstante, esto no quiere decir que deba desecharse. En otros estudios que han tenido acceso a bases de datos más completas (Clarke et al., 2006; Erso, 2006) se logran resultados mejores cuando se consideran las edades de los involucrados en accidentes. Dlvp $p_{i}$ también resultó poco significativa, pues la información disponible para este estudio sobre los datos de accidentes $x_{i}$ en vialidades de primer orden fue limitada y algo similar ocurrió con la variable $D$ int $_{i}$, ya que las $A G E B_{i}$ que tienen una mayor densidad de intersecciones están fuera de la zona de concentración de accidentes.

Posteriormente se realizó una segunda corrida con el resto de los indicadores, lo que arrojó un acumulado hasta el segundo factor de 74. 3\%, que demuestra que esta selección de indicadores es aún mejor.

\subsubsection{Segundo filtro: matriz de correlación de Pearson}

La matriz de correlación de Pearson arrojó datos consistentes con el método de componentes principales. Los indicadores que están altamente relacionados con los accidentes $x_{i}$ son: Demp $_{i}$ (0.607), Dservicios ${ }_{i}$ (0.709), pp60mas

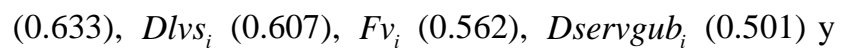
Dues $_{i}$ (0.570). Sin embargo, se debe analizar la colinearidad entre las variables explicativas antes de proceder a seleccionarlas. La matriz de correlación de Pearson muestra alta correlación de la variable $\operatorname{Demp}_{i}$ con las variables Dservicios $_{i}$, Dservgub $_{i}$, Dcomercial $_{i}$, Dindustrial $_{i} \mathrm{y}$ Dues $_{i}$, por lo que se decidió no ingresarlas al modelo para evitar inconsistencias. Las otras variables que se desecharon en esta fase fueron: $i b_{i}$ y pvivcaut ${ }_{i}$, indicadores que tienen representaciones de 0.399 y 0.357 respectivamente. Estas variables están asociadas a la idea de que si hay mejores condiciones de vida las posibilidades de tener un auto serán mayores y también lo será el riesgo de ocurrencia de accidentes. Si bien son indicadores positivos y registran coeficientes de correlación medios (Field, 2005), no son altamente significativos y las pruebas realizadas no indican que aporten un mayor poder explicativo al modelo.

\subsection{Indicadores seleccionados para la instrumentación de los modelos}

Después de la evaluación y depuración de las variables elegidas inicialmente, se decidió utilizar cuatro de ellas para la aplicación de los modelos (ver tabla 2).

\section{ESTRUCTURA GENERAL DE LOS MODELOS}

En este apartado, se muestran tres especificaciones de modelos bajo un enfoque bayesiano (Gregory, 2005), los dos primeros llamados Poisson-Gamma en sus modalidades bivariante y multivariante (Miaou et al., 2003 y Campero, 2011), ambos, permiten calcular la media estimada de accidentes, siguiendo el criterio de conformar diferentes relaciones bivariantes entre los indicadores presentados en la tabla 2, por ejemplo, la relación existente entre "número de accidentes y flujos vehiculares" o "accidentes y densidad de empleo". La aplicacion de varias relaciones, muestra la gran ventaja de comparar y seleccionar el modelo de mayor poder explicativo mediante la bondad de ajuste $R^{2}$. Por otra parte, el tercer modelo llamado Binomial Negativo, refiere el cálculo de la probabilidad de ocurrencia de accidentes viales urbanos (Miranda et al., 2005), este ultimo, permite realizar el análisis de riesgo para la Zona Intrametropolitana de la ciudad de Toluca.

\subsection{Modelo 1 Poisson-Gamma Bivariante}

El primer modelo es conocido como Poisson-Gamma bivariante, su estructura se conforma bajo un enfoque bayesiano mismo que considera una familia de distribuciones Gamma, es decir, la distribución a priori es Gamma, la verosimilitud es Poisson y la distribución a posteriori también es Gamma (Campero, 2011). Este algoritmo permite estimar la media de accidentes anual $\hat{\mu}$ por cada $A G E B_{i}$, los dos insumos considerados son: El registro de datos históricos en cuanto a número de accidentes $x_{i}$ ocurridos en el año 2005 y el flujo vehicular $F v_{i}$ (Bajo el criterio de Quddus), además, la expresión incluye implicitamente un efecto de alatoreidad en el evento y esto se debe a la selección de la distribución Gamma misma que es usada para representar el tiempo aleatorio hasta la ocurrencia de algún otro evento (Gregory, 2005). El modelo se puede observar en la siguiente expresión matemática.

$$
\hat{\mu}=e^{\left(\beta_{1} x_{i}+\ln \left(F v_{i}^{\beta_{2}}\right)+\varepsilon_{i}\right)}
$$

Donde

$e^{\beta_{1} x_{i}}:$ representa los datos históricos de accidentes;

$x_{i}$ : es el número de accidentes tipo colisión ocurridos en la i-ésima $A G E B$;

$\beta_{1}$ : es su correspondiente párametro de estimación; 
$e^{\ln \left(F v_{i}^{\beta_{2}}\right)}:$ define los flujos vehiculares $F v_{i}$ por $A G E B_{\mathrm{i}}$; y análogamente

$\beta_{2}$ : es el parámetro a estimar; y

$e^{\varepsilon_{i}} \sim \operatorname{Gamma}(\alpha, \beta)$ : representa el efecto aleatorio sigue una distribución de probabilidad continua.

En resumen, las variables independientes de este primer modelo toman en cuenta la relación existente entre " $a c$ cidentes y flujo vehicular”, ésta relación es muy significativa para iniciar alguna modelación relativa al tema de accidentes viales y se torna fundamental en este trabajo para interactuar con otras variables en el modelo 3. Para calibrar y sistematizar el modelo se utiliza el programa WinBUGS.14 (Spiegelhalter et al., 2003)

\subsection{Modelo 2 Poisson-Gamma Multivariante}

El segundo modelo es conocido como PoissonGamma multivariante, su estructura se conforma análogamente al modelo 1 (Campero, 2011). Este algoritmo permite estimar la media de accidentes anual $\hat{\mu}$ por cada $A G E B_{i}$, los insumos considerados son: El registro de datos históricos de accidentes $x_{i}$, el flujo vehicular y la densidad de empleo $\operatorname{Demp}_{i}$ (Denue, 2009) y como antes, se considera el efecto de alatoreidad en el evento. Para calibrar y sistematizar este modelo como antes se utiliza el programa WinBUGS.14 (Spiegelhalter et al., 2003) y su estructura ahora considera cuatro factores exponeneciales. El modelo se expresa en la ecuación (4).

$$
\hat{\mu}=e^{\left(\beta_{1} x_{i}+\ln \left(F v_{i}^{\beta_{2}}+D e m p_{i}^{\beta_{3}}\right)+\varepsilon_{i}\right)}
$$

Donde

$e^{\beta_{1} x_{i}}:$ representa los datos históricos de accidentes;

$e^{\ln \left(F v_{i}^{\beta_{2}}\right)}:$ define los flujos vehiculares $F v_{i}$;

$e^{\text {Demp }_{i} \beta_{3}}$ : función que permite estimar el número de empleos por unidad económica por $A G E B_{i}$; y

$e^{\varepsilon_{i}} \sim \operatorname{Gamma}(\alpha, \beta):$ como antes representa el efecto aleatorio.

\subsection{Modelo 3 Probabilístico (Poisson-Gamma o Binomial Negativo).}

El tercer modelo es conocido como Binomial Negativo, su estructura se conforma bajo un enfoque bayesiano (Miranda et al., 2005), esta expresión responde la siguiente incognita: ¿Cuál es la probabilidad condicional de tener algún accidente vial tipo colisión $y_{i}$ en la $A G E B_{i}$, cuando $y_{i}=0,1,2.3, \cdots$ ?, dados los parámetros estimados $\hat{\mu}_{i} \mathrm{y} \varphi$, mismos que representan la media estimada de accidentes y el parámetro de dispersión fijo respectivamente (Miranda et al., 2005; Campero, 2011). Para sistematizar este modelo se utilizó una hoja electrónica Excel. El modelo se puede apreciar en la siguente ecuación.

$$
P\left(y_{i} \mid \hat{\mu}_{i}, \varphi\right)=\frac{\Gamma\left(y_{i}+\varphi\right)}{y_{i} ! \Gamma(\varphi)}\left(\frac{\varphi}{(\varphi+\hat{\mu})}\right)^{\varphi}\left(\frac{\hat{\mu}}{\varphi+\hat{\mu}}\right)^{y_{i}},
$$

con $y_{i}=0,1,2.3, \cdots$

La arquitectura de esta expresión se explica conforme la inferencia bayesiana. De acuerdo a lo establecido por el modelo $P\left(\mu_{i} \mid x_{i}\right) \propto P\left(x_{i} \mid \mu_{i}\right) P\left(\mu_{i}\right)$ se reemplazan las distribuciones de verosimilitud y apriori como se muestra en la ecuación: $P\left(\mu_{i} \mid x_{i}\right) \infty e^{\hat{\mu}\left(n+\beta_{0}\right)} \mu^{n \bar{X}_{i}+\alpha_{0} 1}$

Siguiendo el proceso para normalizar, que consiste en sumar cada probabilidad final y dividir estas entre la suma total $\int_{0}^{\infty}\left(e^{-\mu\left(n+\beta_{0}\right)} \mu^{n \bar{X}_{i}+\alpha_{0}-1}\right) d \mu$, se cumple con el axioma relativo a que la suma de todas las probabilidades sea igual a 1 , como se muestra enseguida.

$$
P\left(\mu \mid x_{i}\right)=\frac{e^{-\mu\left(n+\beta_{0}\right)} \mu^{n \bar{X}_{i}+\alpha_{0}-1}}{\int_{0}^{\infty}\left(e^{-\mu\left(n+\beta_{0}\right)} \mu^{n \bar{X}_{i}+\alpha_{0}-1}\right) d \mu}
$$

Resolviendo la integral de la expresión anterior, la distribución final Gamma queda expresada mediante sus parámetros finales $\left(\alpha_{1}, \beta_{1}\right)$ de la siguiente manera.

$\operatorname{Gamma}\left(\alpha_{1}, \beta_{1}\right)=\left(n \bar{X}+\alpha_{0}, n+\beta_{0}\right)$, donde: los parámetros $\alpha_{0}$ y $\beta_{0}$ se llaman parámetros iniciales.

Estadísticamente hablando, la media y la varianza para la función Gamma quedan expresadas por las siguientes $\quad$ expresiones: $\hat{\mu}=\frac{\alpha_{1}}{\beta_{1}}=\frac{n \bar{X}+\alpha_{0}}{n+\beta_{0}}=\bar{X} \quad$ y $\hat{\sigma}^{2}=\frac{\alpha_{1}}{\beta_{1}^{2}}=\frac{n \bar{X}+\alpha_{0}}{\left(n+\beta_{0}\right)^{2}}=\frac{\bar{X}}{\beta_{1}}$

Bajo el supuesto que $\alpha_{1}$ y $\beta_{1}$ son valores fijos, del despeje de los parámetros $\alpha_{1}=\varphi=n \bar{X}+\alpha_{0}, \beta_{1}=n+\beta_{0} \mathrm{y}$ $\hat{\mu}=\frac{n \bar{X}+\alpha_{0}}{n+\beta_{0}}$ y de la sustitución de ellos en la distribución Binomial Negativa definida por la expresion: $P(Y \mid \hat{\mu}, \varphi)=C_{\alpha-1}^{Y+\alpha-1}\left(\frac{\beta}{\beta+1}\right)^{\alpha}\left(\frac{1}{\beta+1}\right)^{Y}$ se obtiene el modelo probabilístico.

$$
P\left(y_{i} \mid \hat{\mu}_{i}, \varphi\right)=\frac{\Gamma\left(y_{i}+\varphi\right)}{y_{i} ! \Gamma(\varphi)}\left(\frac{\varphi}{(\varphi+\hat{\mu})}\right)^{\varphi}\left(\frac{\hat{\mu}}{\varphi+\hat{\mu}}\right)^{y_{i}},
$$
cuando $y_{i}=0,1,2.3, \cdots$

\section{APLICACIÓN Y RESULTADOS}

\subsection{Aplicación del Modelo 1 Poisson-Gamma Bivariante}

La aplicación de este modelo comprende dos casos: El primero, usa la relación de "accidentes $x_{i}$ con flujos vehiculares" y el segundo, trata la combinación de "accidentes $x_{i}$ con densidad de empleo", ambos casos se ajustan a la siguiente logística: Ingreso de información, calibración del modelo con las primeras 500 iteraciones, continuación del número de iteraciones hasta 10000, mismas que son suficientes y satisfactorias para obtener los valores de los parámetros $\beta_{1}$ y $\beta_{2}$ de manera confiable hasta en un 95\% para las $35 A G E B_{i}$ seleccionadas, consecuentemente, se realiza el cálculo de la media estimada de accidentes $\hat{\mu}_{i}$ como se aprecia en la tabla 3. 
Tabla 3. Parámetros y media estimada usando Poisson Gamma Bivariante

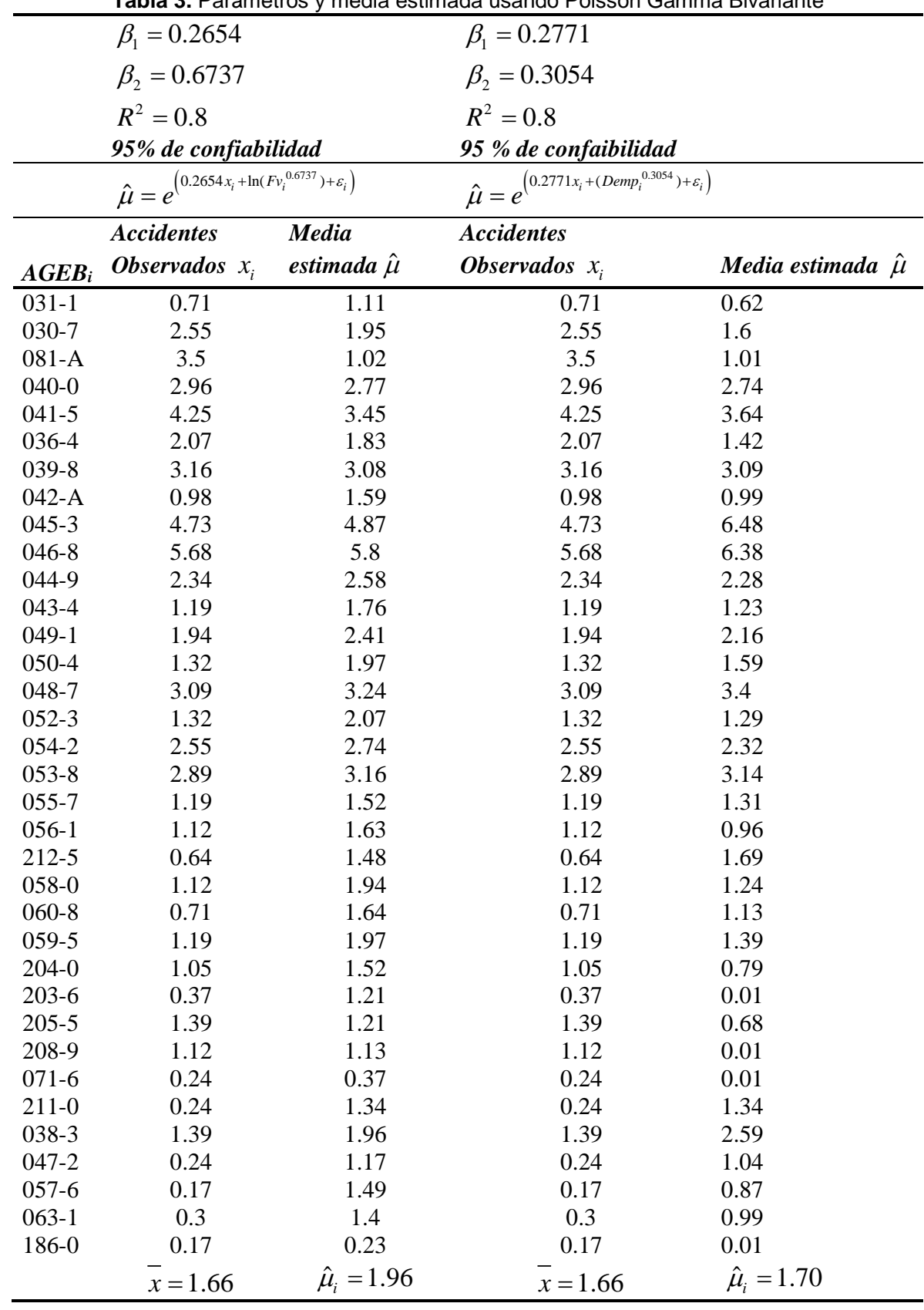

En la tabla anterior, se puede observar la bondad de ajuste de cada regresión de datos con el indicador $R^{2}=0.8$ lo que muestra una alta capacidad explicativa del evento, esta fortaleza indica que las variables propuestas son pertinentes para caracterizar los accidentes viales, sin embargo, existen algunos casos atípicos en ocho AGEB: 081-A, 2125, 060-8, 203-6, 211-0, 047-2, 057-6 y 063-1. Por lo anterior, se decide construir dos gráficas con la intensión de visualizar esquematicamente estos casos atípicos y confirmar el ajuste comentado. La gráfica 1, muestra la comparativa de los datos observados contra los estimados considerando los factores: accidentes $x_{i}$ y flujos vehiculares, ambos favorecen la explicación de un accidente vial.

$\mathrm{Al}$ respecto, se comentan algunos casos ilustrativos de la gráfica 1. Por ejemplo, la $A G E B$ 081-A, registra una importante subestimación de accidentes $x_{i}$, circunstancia que se atribuye al bajo número de autos registrado por vivienda en esa $A G E B$, lo que implica un valor inferido del flujo vehicular bajo, además, por esta $A G E B$ cruza una avenida muy importante llamada Alfredo del Mazo donde la circualción de vehículos es mucho mayor al valor inferido y esto provoca que los resultados del modelo queden muy por debajo de los accidentes observados. Por el contrario, en las otras siete AGEB 212-5, 060-8, 203-6, 211-0, 047-2, 057-6 y 063-1 las regresiones están altamente sobreestimadas, esta situación se atribuye a la falta de información sobre los accidentes que ocurren en Paseo Tollocan, que es una vialidad primaria de jurisdicción estatal, (Sólo se disponía de información en las vialidades de jurisdicción municipal). En resumen, a pesar de estas inverosimilitudes, debe subrayarse que la relación directa entre accidentes y flujo vehicular, reproduce satisfactoriamente los datos observados. Estos resultados son consistentes con los hallazgos de Quddus (2008) y Glaister \& Graham (2005). 

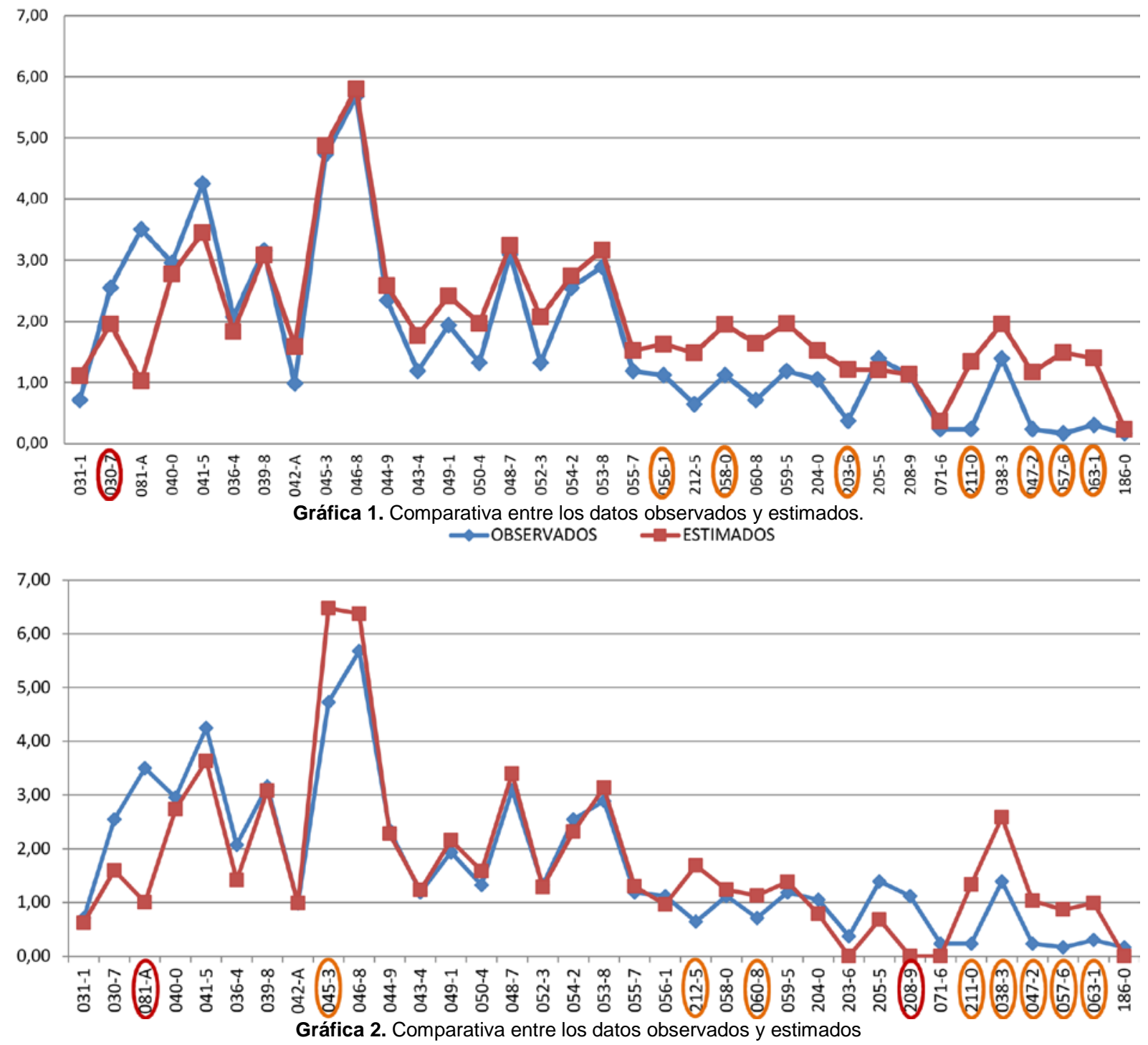

La Gráfica 2, muestra la comparativa de los datos observados contra los estimados considerando la relación entre accidentes y densidad de empleo, ambos favorecen la explicación de un accidente.

Análogamente, en esta gráfica se identifican dos grupos de $A G E B$ que presentan un ajuste atipico. El primer grupo: 081-A y 208-9 se subestiman, por el contrario en el grupo: 045-3, 212-5, 060-8, 211-0, 038-3, 047-2, 057-6 y 063-1, está sobreestimado, este comportamiento se atribuye a: jurisdicciónes, información incompleta y registro de densidades de empleo baja.

\subsubsection{Indicador de efecto indeterminado}

Para otras aplicaciones del Poisson-Gamma Bivariante, es importante reportar la que considera el indicador “proporción de la población mayor de 60 años" que dío un efecto indeterminado como variable independiente. Los resultados de este modelo son menos favorables que las aplicaciones anteriores, pero no son desalentadores. La bondad de ajuste obtenida es $R^{2}=0.6$, sin embargo, se determinó no utilizar la contribución de este factor, al considerar que la limitante es la falta de información sistematizada y validada, dicha información permitiría corroborar que los adultos mayores de 60 años realmente estén implicados en los accidentes viales. Adicionalmente, existe evidencia de que la zona en estudio registra una alta proporción de adultos mayores (Garrocho \& Campos, 2005), por lo que resultaría una falacia considerar este indicador como variable explicativa.

\subsubsection{Indicador con bajo poder explicativo}

En otro contexto de aplicación hacia el modelo Poisson-Gamma bivariante, la relación existente entre "accidentes y densidad de vías secundarias”, deriva que esta prueba detecta un desplome en la bondad de ajuste de $R^{2}=0.1$ lo que indica un bajo poder explicativo en el tema accidentes $x_{i}$.

En resumen, se puede concluir que las variables para conformar un modelo multivariante son: "Registro de accidentes $x_{i}$ historico, flujo vehicular estimado y densidad de empleo", se espera que este conjunto de indicadores contribuya a la caracterización del evento estadístico llamado accidente $x_{i}$ y con ello complementar nuestro sistema de información geográfica (SIG) que permita mostrar zonas potencialmente peligrosas, de esta manera, se podrá contribuir en la toma de desiciones en cuanto al tema de seguridad vial se refiere. 
Tabla 4. Parámetros y media estimada utilizando Poisson-Gamma Multivariante

\begin{tabular}{|c|c|c|c|c|}
\hline & & $\begin{array}{l}\beta_{1}=-0.1161 \\
\beta_{2}=0.7075 \\
\beta_{3}=0.7948 \\
R^{2}=0.6\end{array}$ & & \\
\hline \multicolumn{5}{|c|}{$\hat{\mu}=e^{\left(-0.1161 x_{i}+\ln \left(F v_{i}^{0.7075}\right)+\text { Demp }_{i}^{0.7948}+\varepsilon_{i}\right)}$} \\
\hline$\overline{A G E B_{i}}$ & Observado & Estimado & $S D$ & MC error \\
\hline 031-1 & 0.71 & 0.8374 & 0.05874 & 0.001669 \\
\hline 040-0 & 2.96 & 1.479 & 0.3017 & 0.007172 \\
\hline 036-4 & 2.07 & 0.8664 & 0.1551 & 0.004495 \\
\hline 039-8 & 3.16 & 1.933 & 0.3879 & 0.00786 \\
\hline 042-A & 0.98 & 1.206 & 0.09337 & 0.002589 \\
\hline $045-3$ & 4.73 & 5.767 & 1.624 & 0.02273 \\
\hline 044-9 & 2.34 & 2.076 & 0.295 & 0.005034 \\
\hline 043-4 & 1.19 & 1.378 & 0.1178 & 0.002898 \\
\hline 049-1 & 1.94 & 2.854 & 0.3486 & 0.004713 \\
\hline 050-4 & 1.32 & 2.041 & 0.1754 & 0.002483 \\
\hline 048-7 & 3.09 & 3.628 & 0.6617 & 0.008432 \\
\hline 052-3 & 1.32 & 1.713 & 0.1647 & 0.003649 \\
\hline $054-2$ & 2.55 & 1.899 & 0.3072 & 0.006213 \\
\hline 053-8 & 2.89 & 3.901 & 0.6815 & 0.009081 \\
\hline $055-7$ & 1.19 & 1.068 & 0.0983 & 0.002701 \\
\hline 056-1 & 1.12 & 1.141 & 0.1037 & 0.002975 \\
\hline 060-8 & 0.71 & 1.673 & 0.1032 & 0.002015 \\
\hline $059-5$ & 1.19 & 1.923 & 0.1609 & 0.002746 \\
\hline 204-0 & 1.05 & 1.051 & 0.09337 & 0.002765 \\
\hline 203-6 & 0.37 & 1.059 & 0.03595 & 0.001094 \\
\hline $205-5$ & 1.39 & 0.7014 & 0.09648 & 0.002713 \\
\hline $208-9$ & 1.12 & 0.7138 & 0.08283 & 0.002347 \\
\hline 071-6 & 0.24 & 0.2789 & 0.07394 & 0.001893 \\
\hline 047-2 & 0.24 & 1.187 & 0.02337 & 4.27E-04 \\
\hline 063-1 & 0.3 & 1.574 & 0.08115 & 0.001925 \\
\hline \multirow[t]{2}{*}{$186-0$} & 0.17 & 0.04337 & 0.02602 & 6.98E-04 \\
\hline & $\bar{x}=1.55$ & $\hat{\mu}_{i}=1.69$ & & \\
\hline
\end{tabular}

\subsection{Aplicación del Modelo 2 Poisson-Gamma Multivariante}

La aplicación de este modelo se ajusta a la relación existente entre "accidentes $x_{i}$, flujos vehiculares $y$ densidad de empleo”, conservando la misma logística que se mencionó en la primera especificación, además, se aplica el criterio de eliminar las nueve $A G E B$ que registraron desajuste estadístico entre lo calculado y lo observado, reduciendo el análisis a $26 A G E B_{i}$. Esto con la finalidad de lograr una mejor regresión. Los resultados obtenidos se presentan en la tabla 4.

Tomando en cuenta los resultados de la tabla 4 , la modelación indica una bondad de ajuste $R^{2}=0.6$, al respecto se comenta lo siguiente: Se considera que la caracterización de los accidentes $x_{i}$, dado el conjunto de variables seleccionadas de mayor significancia, es bastante acertiva, puesto que un modelo que presente un $R^{2}$ cercano a la unidad, puede resultar falso al considerar minimas variables explicativas, en nuestro caso, el resultado es lógico y favorecedor tomando en cuenta, la contribución de frecuencia de accidentes $x_{i}$, flujo de tráfico urbano y que decir de las actividades laborales en estas zonas que generan un aumento considerable en desplazamientos, es decir, estos ele- mentos del entorno físico urbano asemeja una mejor realidad para la explicación de la ocurrencia de un accidente vial $x_{i}$.

La desición que se tomó de eliminar las $A G E B_{i}$ outliers logra la mejora del indicador de ajuste: originalmente la bondad de ajuste con $35 A G E B_{i}$ fue $R^{2}=0.4$ y con $26 A G E B_{i}$ se logró una bondad de ajuste de $R^{2}=0.6$ lo que significa que el modelo ajusta los datos observados en más de $60 \%$.

La gráfica 3, muestra el ajuste entre los accidentes observados y la media estimada de la muestra depurada para la zona intrametropolítana de la ciudad de Toluca.

No obstante, en la gráfica 3 se observan algunos casos atípicos. Por ejemplo, la ocurrencia de accidentes en las AGEB 040-0 y 036-4 está subestimado. La AGEB 040-0 tiene una de le las densidades de empleo más altas, pero el flujo vehicular inferido no es alto. Esto puede significar que nuestra forma de inferir los flujos vehiculares esté generando ciertas inexactitudes en el modelo. La AGEB 036-4 presenta un flujo vehicular medio, sin embargo, la densidad de empleo es bastante baja lo que ocasiona el sesgo hacia la subestimación de la media de accidentes.

Por otro lado, la ocurrencia de accidentes en las AGEB 060-8, 059-5, 203-6, 047-2 y 063-1 está sobreestimado, en parte también por la manera como se infirieron los flujos vehiculares, pero además porque el dato observado padece un subregistro, puesto que sólo se consideraron vías 


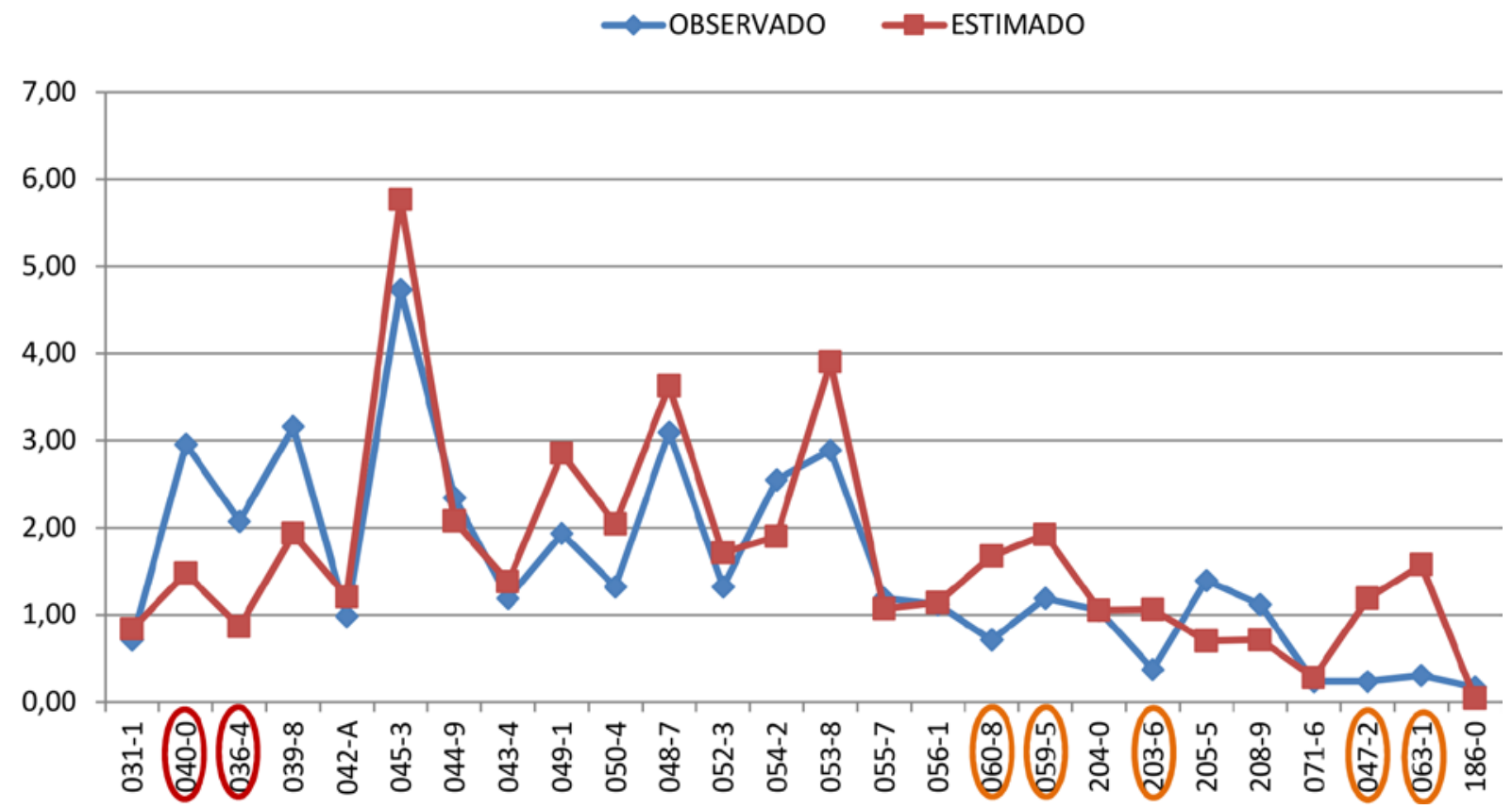

Gráfica 3. Comparativa entre los datos observados y estimados

Tabla 5. Cálculo de probabilidades con el modelo Binomial Negativo

\begin{tabular}{|c|c|c|c|c|c|}
\hline \multicolumn{3}{|c|}{$P\left(y_{i} \mid \hat{\mu}_{i}, \varphi\right)=\frac{\Gamma\left(y_{i}+\varphi\right)}{y_{i} ! \Gamma(\varphi)}($} & $\left.\frac{\varphi}{+\hat{\mu})}\right)^{\varphi}$ & $\left.\frac{\hat{\mu}}{\varphi+\hat{\mu}}\right)^{y_{i}}$ & \multirow[b]{2}{*}{ \%Probabilidad } \\
\hline$A G E B$ & Observado & Estimado & ProbBN & Normalizado & \\
\hline $031-1$ & 0.71 & 0.8374 & 0.02 & 0.01 & 0.75 \\
\hline 040-0 & 2.96 & 1.479 & 0.01 & 0.00 & 0.46 \\
\hline $036-4$ & 2.07 & 0.8664 & 0.20 & 0.08 & 8.08 \\
\hline 039-8 & 3.16 & 1.933 & 0.09 & 0.03 & 3.44 \\
\hline 042-A & 0.98 & 1.206 & 0.01 & 0.00 & 0.31 \\
\hline $045-3$ & 4.73 & 5.767 & 0.01 & 0.00 & 0.24 \\
\hline 044-9 & 2.34 & 2.076 & 0.08 & 0.03 & 3.33 \\
\hline $043-4$ & 1.19 & 1.378 & 0.17 & 0.07 & 6.67 \\
\hline 049-1 & 1.94 & 2.854 & 0.01 & 0.01 & 0.58 \\
\hline $050-4$ & 1.32 & 2.041 & 0.11 & 0.04 & 4.28 \\
\hline 048-7 & 3.09 & 3.628 & 0.11 & 0.04 & 4.29 \\
\hline $052-3$ & 1.32 & 1.713 & 0.11 & 0.04 & 4.28 \\
\hline 054-2 & 2.55 & 1.899 & 0.04 & 0.02 & 1.72 \\
\hline 053-8 & 2.89 & 3.901 & 0.01 & 0.01 & 0.57 \\
\hline 055-7 & 1.19 & 1.068 & 0.17 & 0.07 & 6.67 \\
\hline 056-1 & 1.12 & 1.141 & 0.21 & 0.08 & 8.32 \\
\hline 060-8 & 0.71 & 1.673 & 0.02 & 0.01 & 0.75 \\
\hline $059-5$ & 1.19 & 1.923 & 0.17 & 0.07 & 6.67 \\
\hline 204-0 & 1.05 & 1.051 & 0.26 & 0.10 & 10.38 \\
\hline $203-6$ & 0.37 & 1.059 & 0.06 & 0.02 & 2.26 \\
\hline $205-5$ & 1.39 & 0.7014 & 0.09 & 0.03 & 3.43 \\
\hline $208-9$ & 1.12 & 0.7138 & 0.21 & 0.08 & 8.32 \\
\hline 071-6 & 0.24 & 0.2789 & 0.09 & 0.04 & 3.51 \\
\hline 047-2 & 0.24 & 1.187 & 0.09 & 0.04 & 3.51 \\
\hline 063-1 & 0.30 & 1.574 & 0.07 & 0.03 & 2.82 \\
\hline $186-0$ & 0.17 & 0.04337 & 0.11 & 0.04 & 4.39 \\
\hline & & Total & 2.52 & 1 & 100 \\
\hline
\end{tabular}

de jurisdicción municipal (subrayamos que no fue posible obtener información para las vías de jurisdicción estatal).

Estos resultados atípicos lejos de mostrar errores en el modelo, lo corroboran, pues las $A G E B_{i}$ que se manifiestan atípicas registran en la realidad características peculiares que no fue posible incluir en el modelo por falta de información más detallada. Incluso, a pesar de haber inferido de manera indirecta un dato tan importante como el flujo vehicular, los modelos tienen indicadores estadísticos que demuestran su fortaleza más allá de toda duda.

\subsection{Aplicación del Modelo 3 Probabilistico Binomial Negativo}

Para la aplicación del modelo Binomial Negativo, se siguen las siguientes etapas: La primera, consiste en calcular los parámetros de la distribución 


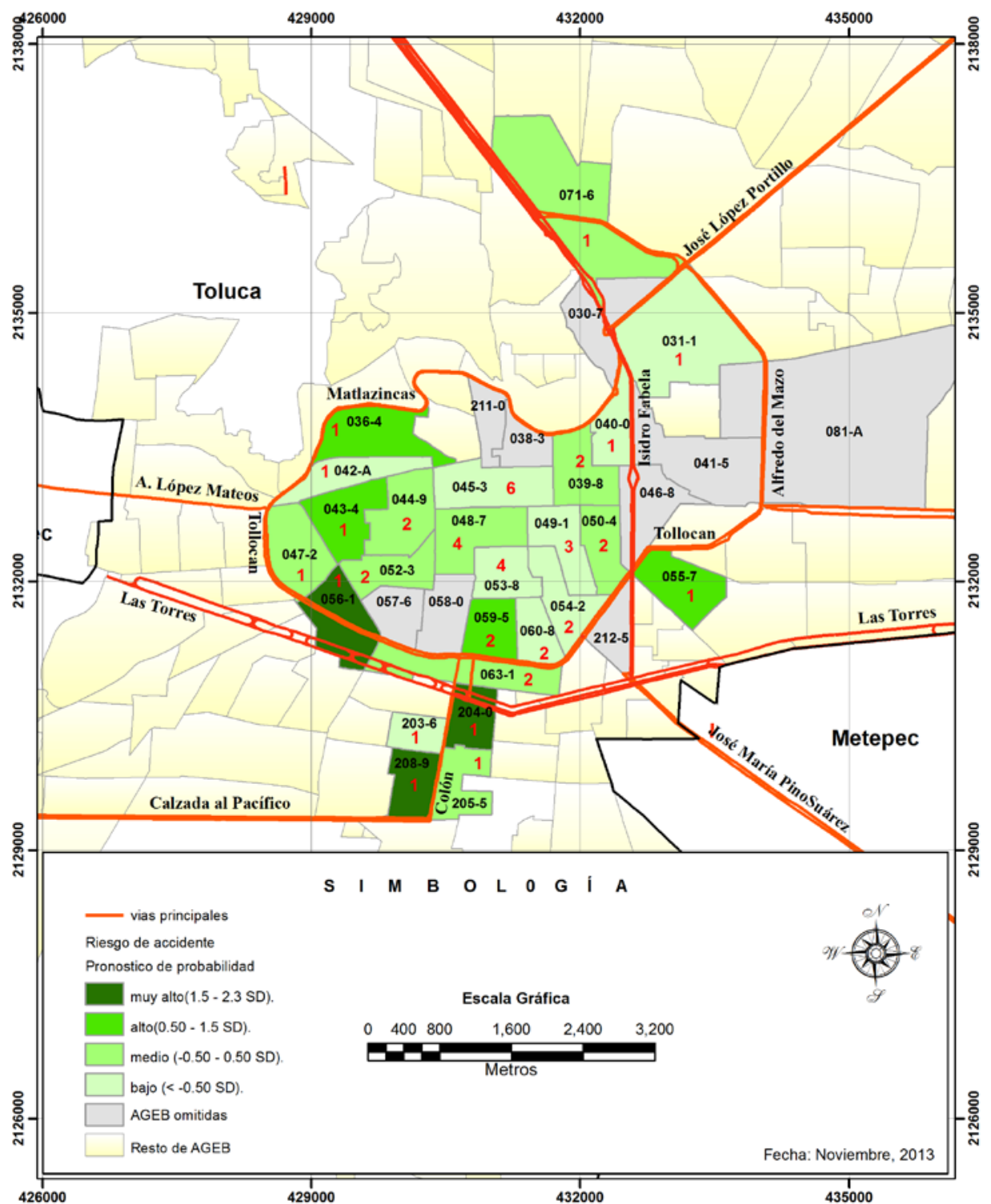

Figura 3. Zonas de riesgo para 26 AGEB en la zona intrametropolitana de Toluca

$\operatorname{Gamma}\left(\alpha_{0}=2.4, \beta_{0}=1.2\right)$ lo que permite calcular la distribución final $\operatorname{Gamma}\left(\alpha_{1}=42, \beta_{1}=30\right)$, esta distribución final tiene: media $\hat{\mu}=\frac{\alpha_{1}}{\beta_{1}}=\frac{42}{30}=1.40$. Como segunda etapa se calculan los parámetros $\hat{\mu}$ y $\varphi, \hat{\mu}=1.40$, $\alpha_{1}=\varphi=42$ parámetros a utilizar en este modelo. Los resultados de esta aplicación se presentan en la tabla 5.

En la tabla anterior se aprecia lo siguiente: Por ejemplo, la aplicación del modelo 3 indica que el grupo de AGEB: 204-0, 056-1 y 208-9 pueden registrar hasta un (1) accidente $x_{i}$ con una probabilidad de $10.34 \%$ y $8.32 \%$ respectivamente, en cambio las AGEB: 045-3 y 053-8 pueden registrar hasta seis (6) y cuatro (4) accidentes $x_{i}$ por año con una probabilidad menor de $0.24 \%$ y $0.57 \%$. En general, la accidentalidad varía desde 1 hasta 6 accidentes $x_{i}$ por $A G E B$. Usualmente la probabilidad de ocurrencia registra una relación inversa con el número de accidentes $x_{i}$, en otras palabras, es común que en el territorio exista más probabilidad de que ocurra un número reducido de accidentes $x_{i}$ y menos probabilidad de que suceda un número elevado de accidentes $x_{i}$.
Finalmente, se presenta la figura 3 de riesgo de accidentes $x_{i}$ zonificado por $A G E B$, las directrices empleadas son las siguientes: Se ingresaron las estimaciones de accidentes $x_{i}$ en el SIG, se obtiene la distribución espacial por $A G E B$ y se realiza una graduación por color. Es relevante comentar acerca del alto riesgo detectado en vialidades primarias y secundarias, índice que se atribuye a las $A G E B$ que registran elevada densidad de empleo. Por ejemplo: las AGEB, 036-4, 043-4, 056-1, 204-0, 208-9 y 055-7 muestran que se puede presentar almenos 1 accidente vial tipo colisión con una probabilidad de ocurrencia alta con porcentajes de $8.1 \%, 6.7 \%, 8.3 \%, 10.4 \%, 8.3,6.7 \%$ respectivamente. En el caso específico de la AGEB 045-3 el análisis indica que se pueden presentar hasta 6 accidentes $x_{i}$, sin embargo, la probabilidad de que esto ocurra es muy baja de $0.24 \%$.

\section{CONCLUSIONES}

Esta investigación se fundamentó en estadística clásica, inferencia bayesiana y uso de SIG. Diversos teóricos han utilizado recientemente estas técnicas para la modelación y caracterización de los datos de accidentes de tránsito. 
Varios estudios, incluyendo este, coinciden en que las características del espacio y ciertos elementos de la estructura urbana tienen un efecto significativo en los eventos de accidentalidad (e.g. Lord et al., 2005; Miaou \& Jin, 2005).

Este trabajo no sólo presenta modelos capaces de pronosticar con un alto grado de exactitud la magnitud y probabilidad de ocurrencia de accidentes $x_{i}$ en la zona intrametropolitana de Toluca, sino que devela además hasta qué punto la población, el empleo, los flujos vehiculares y la naturaleza del desarrollo urbano se relacionan con los accidentes $x_{i}$ en una ciudad mexicana e identifica áreas o zonas con alto riesgo de accidentes $x_{i}$. Estos resultados son insumos clave para el diseño de políticas públicas cuyo propósito central sea la prevención de accidentes.

En la zona intrametropolítana de Toluca las AGEB que presentan mayor densidad de flujo vehicular y empleo tienen mayor probabilidad de experimentar accidentes $x_{i}$. La densidad de viajes y movimientos depende del empleo, que es mayor y tiende a concentrarse en los centros tradicionales de negocios de las ciudades mexicanas, lo que incrementa el riesgo de accidentes $x_{i}$. Esto es consistente con los hallazgos de Fuentes \& Hernández (2009) para otras ciudades mexicanas. Por tanto, las zonas que son generadoras de flujos continuos de tránsito (debido a la localización de actividades económicas), deben ser estratégicas en el diseño de políticas públicas que busquen reducir los riesgos que produce la movilidad al interior de la ciudad.

En el área de estudio los indicadores relacionados con fuertes densidades de población y viviendas no resultaron significativos en términos del riesgo de sufrir accidentes $X_{i}$. En cambio, las zonas con baja densidad de población (i.e. intensa mezcla de usos de suelo) tienen mayor probabilidad de sufrir accidentes de tránsito, debido a que comparten el uso de suelo con otras actividades económicas (comercio, servicios y pequeña industria), lo que genera una mayor demanda de viajes y un entorno más riesgoso para los automovilistas.

\section{REFERENCIAS}

Aguero-Valverde, J. \& P. P. Jovanis, (2006) Spatial analysis of fatal and injury crashes in Pennsylvania. Journal of Accident Analysis and Prevention, v.38, p.618-625:

DOI:10.1016/j.aap.2005.12.006

Alcántara, V. E., (2010) “Análisis de movilidad urbana. Espacio, medio ambiente y equidad”. Ed. CAF. Bogotá, Colombia. p. 204.

Boher, J. H. I. (2006). Pacto por la Movilidad. La seguridad vial en Barcelona, España. Ayuntamiento de Barcelona. p. 48. w110.bcn.cat/fitxers/mobilitat/pacte/pacte8e.380.pdf.

Campero, A. C., (2011). Pronóstico de accidentes viales en intersecciones de la ciudad de Toluca utilizando enfoque Bayesiano. (Tesis inédita de maestría). Universidad Autónoma del Estado de México, México.

Campos, A. J., (2009). La Geografía de la marginación: enfoque conceptual y metodológico alternativo para el caso de México. (Tesis inédita de doctorado). Universidad Nacional Autónoma de México, México.

Consejo Nacional de Población CONAPO, (2005). Indice de marginación a nivel localidad (Anexo C Metodología de estimación del índice de marginación). SEGOB. D.F., México. www.conapo.gob.mx/es/CONAPO/Indice_de_marginacion_a_ni vel_localidad_2005..

Clarke, D., P. Ward, C. Bartle, W. Truman, (2006), Young driver accidents in UK: the influence of age, experience, and time of day. Journal of Accident Analysis and Prevention, v.38 p. 871878. DOI:10.1016/j.aap.2006.02.013

Clifton, K., J. Carolina, V. Burnier, \& A. Gulsah, (2009). Severity of injury resulting from pedestrian-vehicle crashes: What can we learn from examining the built environment. Journal Transportation Research Part D, v.14, p. 425-436. DOI:10.1016/j.trd.2009.01.001

Chueca, G. F., (1997). Breve historia del urbanismo. España: Editorial Alianza.

Christie, N. (2000). The high risk child pedestrian: socioeconomic and environmental factors in their accidents. PR 117. Journal TRL. p. 33.

De Mattos, (2002). Transformación de las ciudades latinoamericanas: ¿ ¿Impactos de la globalización? EURE, v.28 p. $5-10$.

European Road Safety Observatory (2006). Novice Drivers. retrieved april 10, 2007 from www.erso.eu.

Field A., (2005). Discovering statistics using SPSS. Londres, UK: Sage.

Fuentes, F. C. M. \& H. V. Hernández (2009). La estructura espacial urbana y la incidencia de accidentes de tránsito en Tijuana, Baja California (2003-2004). Redalyc Frontera Norte 2009, v. 21 p.109-138: Disponible en:

www.redalyc.uaemex.mx/src/inicio/ArtPdfRed.jsp?iCve=136120 40005 .

Garrocho, C. \& J. A.Campos, (2005). La población adulta mayor en el área metropolitana de Toluca, 1990-2000. Papeles de Población, Año 11, v. 45, p. 71-106: Disponible en: www.redalyc.uaemex.mx/pdf/112/11204505.pdf.

Garrocho, C. \& Z. Flores, (2009). Delimitación del centro tradicional de comercio y servicios del Área Metropolitana de Toluca. Papeles de Población, v.15, p.233-274.

Garrocho, R. C., (2013). “Dinámica de las ciudades de México en el siglo XXI. Cinco vectores clave para el desarrollo sostenible”. México, ONU: Fondo de Población (UNFPA)Conapo-El Colegio Mexiquense, México.

Garza, N., (2009). Dilema económico-ambiental del urbanismo en ciudades latinoamericanas. Investigación y Desarrollo, v. 17, p. 288-327. Universidad del Norte Barranquilla, Colombia. Disponible en: www.redalyc.org/pdf/268/26816278004.pdf.

Graham, D., S. Glaister, \& R. Anderson, (2005). The effects of area deprivation on the incidence of child and adult pedestrian casualties in England. Journal of Accident Analysis and Prevention v.37, p.125-135. DOI: 10.1016/j.aap.2004.07.002

Graizbord, B., (2008). “Geografia del Transporte en el Area Metropolitana de la Ciudad de Mexico”. Cedua, El Colegio de México. D.F., México. p. 386.

Gregory, P. C., (2005). "Bayesian logical data analysis for the phisycal sciences. A comparative approach with mathemática support”. Cambrige University Press. New York, United States. p. 468. www.cambridg e.org /9780521841504 
Hadayeghi, A., A.S. Shalaby, \& B.N. Persaud. (2003).

Macrolevel accident prediction models for evaluating safety of urban transportation systems. Journal of Safety and Human performance, v.1840 p.87-95:

http://trb.metapress.com/content/av604731263mn076/.

Instituto Nacional de Estadistica y Geografía, INEGI, (2009). Directorio Estadístico Nacional de Unidades Económicas.

México.

http://www3.inegi.org.mx/sistemas/mapa/denue/default.aspx.

Instituto Nacional de Estadistica y Geografía, INEGI, (2005).

Censos y Conteos de población y vivienda. Conteos de Población y Vivienda 2005. México.

www.inegi.org.mx/est/contenidos/proyectos/ccpv/cpv2005/.

Jones, A. P., R. Haynes, V. Kennedy, I. M. Harvey, T. Jewell, \& D. Lea, (2008). Geographical variations in mortality and morbidity from road traffic accidents in England and Wales. Journal of Health \& place, v.14, p. 519-535.

DOI:10.1016/j.healthplace.2007.10.001.

Kim, K., I.M. Brunner \& E.Y. Yamashita, (2006). The influence of land use, population, employment and economic activity on Accidents. Journal of Safety and Human performance, v.1953, p. 56-64, TRB, National Research Council, Washington DC: Disponible en:

www.trb.metapress.com/content/y188nx845j77566v/.

Lord, D., S. M. Washington, \& J. N. Ivan, (2005). Poissongamma and zero inflacted regression models of motor vehicle crashes: balancing statistical fit and theory. Journal of the transportation research board. v.37, p. 35-46.

Miaou, S. \& D. Lord, (2003). Modeling traffic crash-flow relationship for intersections. Dispersion parameter, functional form, and Bayes versus empirical Bayes methods. Journal of Transportation Research Record v. 1840, p. 31-41.

DOI:10.3141/1840-04

Miaou, S. \& J. Jin (2005). Bayessian ranking of sites for engineering safety improvements: Decision parameter, treatability concept, statistical criterion, and spatial dependence. Journal of Accident Analysis and Prevention. v. 37, p. 699-720. DOI:10.1016/j.aap.2005.03.012

Miranda, M. L. F., L. Fu, F. F. Saccomanno, \& A. Labbe, (2005). Alternative risk models for ranking locations for safety improvement. Journal of the Transportation Research Board No. 1908, Transportation Research Board of the National Academies, Washington, D.C., p. 1-8. DOI: 10.3141/1908-01

Negrete, P. M., (2002). Las metrópolis latinoamericanas en la red mundial de ciudades: ¿megaciudades o ciudades globales? Edita Instituto Juan de Herrera 4. 28040 MADRID. ESPAÑA. p. 10. http://habitat.aq.upm.es/boletin/n22/ampez.html.

Noland, B. R. \& M. A. Quddus, (2003). A spatially disaggregate analysis of road casualties in England. Journal of Accident Analysis and Prevention. v. 36 p. 973-984. DOI:

10.1016/j.aap.2003.11.001.

Peden, M., R. Scurfield., D. Sleet, Mohan, D., A. Adnan, Hyder, E. Jarawan \& C. Mathers, (2004). World report on road traffic injury prevention. Geneva: World Health organization.

Pulugurtha, S. S., V. R. Duddu, \& Y. Kotagiri, (2012). Traffic analysis zone level crash estimation models based on land use characteristics. Journal of Accident Analysis and Prevention. v. 50 p. 678-687 DOI: 10.1016/j.aap.2012.06.016.
Quddus, M. A., (2008). Modelling area-wide count outcomes with spatial correlation and heterogeneity: An analysis of London crash data. Journal of Accident Analysis and Prevention. v. 40 p. 1486-1497. DOI:10.1016/j.aap.2008.03.009

Rodríguez F. G. (2012). Sistema de consulta Geoelectoral para el Estado de México. Periodo 1990-2009. (Tesis inédita de maestría), Universidad Autónoma del Estado de México, México.

Siddiqui, Ch., M. Abdel-Aty, K. Choi, (2012). Macroscopic spatial analysis of pedestrian and bicycle crashes. Journal of Accident Analysis and Prevention. v. 45, p. 382-391. DOI:10.1016/j.aap.2011.08.003

Spiegelhalter D., A. Thomas, Best Nicky \& D. Lunn. (2003). “Manual. WinBUGS User Manual. Versión 1.4". MRC

Biostatistics Unit, Intitute of Public Health, Robinson Way, Cambridge CB2 2SR, UK. Departament of Epydemiology \& Public Health, Imperial College School of Medicine, Norfolk Place, London W2 1PG, UK. 